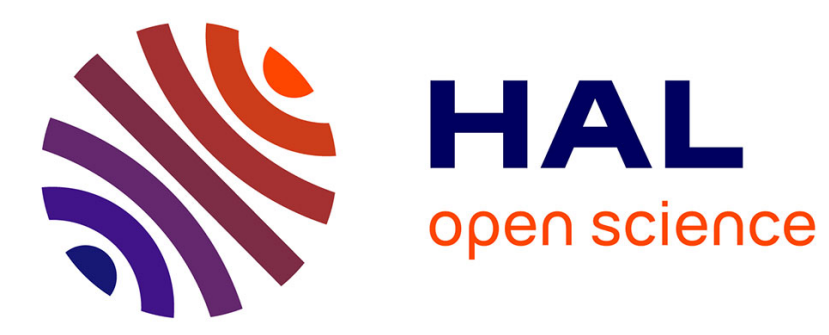

\title{
High gain observer based on-line rotor and stator resistances estimation for IMs
}

Salim Hadj Saïd, Faouzi Mimouni, Faouzi M'Sahli, Mondher Farza

\section{To cite this version:}

Salim Hadj Saïd, Faouzi Mimouni, Faouzi M'Sahli, Mondher Farza. High gain observer based on-line rotor and stator resistances estimation for IMs. Simulation Modelling Practice and Theory, 2010, pp.1927-1932. 10.1016/j.simpat.2011.03.006 . hal-01059312

\section{HAL Id: hal-01059312 \\ https://hal.science/hal-01059312}

Submitted on 29 Aug 2014

HAL is a multi-disciplinary open access archive for the deposit and dissemination of scientific research documents, whether they are published or not. The documents may come from teaching and research institutions in France or abroad, or from public or private research centers.
L'archive ouverte pluridisciplinaire HAL, est destinée au dépôt et à la diffusion de documents scientifiques de niveau recherche, publiés ou non, émanant des établissements d'enseignement et de recherche français ou étrangers, des laboratoires publics ou privés. 


\title{
High gain observer based on-line rotor and stator resistances estimation for IMs
}

\author{
S. Hadj Saïd ${ }^{\mathrm{a}, *}$, M.F. Mimouni ${ }^{\mathrm{a}}$, F. M'Sahli ${ }^{\mathrm{a}}$, M. Farza ${ }^{\mathrm{b}}$ \\ ${ }^{a}$ Department of Electrical Engineering, Monastir Engineering School, Road Ibn Eljazzar, 5019 Monastir, Tunisia \\ ${ }^{\mathrm{b}}$ GREYC, UMR 6072 CNRS, Universite de Caen, 6 Bd Marechal Juin, 14050 Caen Cedex, France
}

Keywords:

Parameters estimation

States estimation

Adaptive observer

Induction motors

\begin{abstract}
A B S T R A C T
One of the main challenging issues in induction motor drives is the lack of knowledge about the actual values of some critical parameters, such as rotor and stator resistances which are subject to large variations during operation. Such problem is difficult to resolve due to the strong interconnection between states and parameters in the nonlinear motor model, besides the unavailability of both rotor flux and load torque. In the spirit to accurately follow the on-line machine variables, this paper focuses on the simultaneous estimation of internal states and time varying parameters. Especially, a new identification scheme for rotor resistance and/or stator resistance is introduced. In the aim of decoupling the unknown electrical parameters, we adopt a mild change of coordinates that allows to easily design a two-stage of high gain observer. The simplicity of presented procedures and the efficiency for real time computation constitute both main features of the proposed approach. Moreover, possible exploitation of our algorithm in the fault detection issue is discussed through a simulation of an abrupt rotor short-circuit.
\end{abstract}

\section{Introduction}

In high performance control of induction motors (IM), it is often important to follow time evolution of both machine parameters and internal state variables. Nonetheless, installation of physical sensors for such applications leads to increase cost, reliability and sensitivity to electromagnetic noise [11,12]. In addition, the lack of knowledge of both rotor and stator resistances which are subjected to large variations during operations, exhibits a poor control performances and in general increases the consummation energy. All this gives a strong motivation towards the development of adaptive observer schemes for simultaneous estimation of parameters and state variables.

For the parameters mismatch, it is essential to know that in addition to the load torque, the temperature and the frequency dependent variation of the stator and rotor resistances. In particular, the variation of the rotor resistance due to temperature can increase by an order of $100 \%$, which involves significant undesirable effects on the vector control algorithm [19]. In other hand, the stator currents strongly affects the stator resistance due to the ohmic losses in the stator winding, which presents a serious hindrance when a vector controlled drive operates at low speed regions [6]. Therefore, many research efforts have been made to alleviate these performances degradation by designing new algorithms that allow an estimation of the rotor and/or stator resistances during operation $[3,4,6,14,19,21,22,17]$. For a conjoint rotor and stator resistances identification scheme, it is suggested in [13], an intricate stator current observer such that its estimation error is guaranteed to converge in order to find the stator resistance. The estimator employed in [18] is able to follow the actual values of both

\footnotetext{
* Corresponding author. Tel.: +216 22731759.

E-mail address: salim.hadjsaid@issig.rnu.tn (S.H. Saïd).
} 
resistances very efficiently, but only when the load torque is assumed constant. The immersion technique based forgetting factor observer is investigated in [2]. The authors propose to extend IM model to obtain a representation which is affine w.r.t. variables to be estimated. Nevertheless, this strategy remains inapplicable in real time implementation because its high computation burden. A development of switching extended Kalman filter (EKF) for rotor and stator resistances in speed sensorless control of IM is introduced in [5]. Such design as common EKF alternatives, suffers from the lack of stability results in various operating ranges and also the requirement of high computational capacities.

Some other contributions based on the model reference adaptive system (MRAS) methods are proposed in [6,15] and [16]. Besides the persistent excitation requirement, these sensorless algorithms usually failed at zero rotor speed with nominal load torque as reported in [21]. An independent line of research was focused in the design of adaptive observers in [7] and [1], for respectively continuous and continuous-discrete cases. A main drawback of these alternatives that only constant unknown parameters can be evaluated.

In the present work, we shall detail a new estimation algorithms of rotor resistance and/or stator resistance for IM. Indeed, under a mild change of coordinates, two-stage of high gain observer (HGO) are used to accurately determine on-line the internal state variables and the time varying parameters. The paper is organized as follows: in Section 2, the mathematical model of IM is recalled. We describe in Section 3 the procedure of designing a suitable HGO corresponding to the particular class of nonlinear systems in which the IM model should be transformed. In Section 4, four schemes for the real-time estimation of IM variables are introduced with relevant simulation results. The first and second schemes are for separate identification of the rotor and stator resistances, while the third scheme performs simultaneous estimation of these two parameters and the fourth one deals with the rotor resistance estimation in the sensorless case. Concluding remarks are given at the final section.

\section{The IM model and problem statement}

Assuming linear magnetic circuits, the dynamics of a balanced non-saturated induction motor in a fixed reference frame attached to the stator are given in a condensed form as follows:

$$
\left\{\begin{array}{l}
i=K A\left(\omega, \alpha_{r}\right) \Psi-\gamma\left(\alpha_{r}, \alpha_{s}\right) l+\frac{1}{\sigma L_{s}} u \\
\dot{\Psi}=-A\left(\omega, \alpha_{r}\right) \Psi+M \alpha_{r} l \\
\dot{\omega}=\frac{p M}{J L_{r}} l^{T} J_{2} \Psi-\frac{1}{J} T_{L} \\
\dot{T}_{L}=\varepsilon_{T_{L}} \\
y=l
\end{array}\right.
$$

in which $\omega$ is the rotor speed, $\Psi=\left[\Psi_{\alpha r}, \Psi_{\beta r}\right]^{T}$ is the rotor flux vector, $l=\left[l_{\alpha s}, l_{\beta s}\right]^{T}$ and $u=\left[u_{\alpha s}, u_{\beta s}\right]^{T}$ are the stator current and voltage vectors in a fixed reference attached to the stator. The model parameters are: rotor moment of inertia $J$, rotor and stator winding's resistances $\left(R_{r}, R_{S}\right)$ and inductances $\left(L_{r}, L_{S}\right)$, mutual inductance $M$ and load torque $T_{L}$. To simplify notations we use the reparametrization: $K=\frac{M}{\sigma L_{r} L_{s}}, \sigma=1-\frac{M^{2}}{L_{L} L_{s}}, \gamma\left(\alpha_{r}, \alpha_{s}\right)=\frac{\alpha_{s}}{\sigma}+M \alpha_{r} K, A\left(\omega, \alpha_{r}\right)=\alpha_{r} I_{2}-p \omega J_{2}$, with $\alpha_{r}=R_{r} / L_{r}, \alpha_{s}=R_{s} / L_{s}$, $I_{2}$ is the 2-dimensional identity matrix and $J_{2}=[0-1 ; 10]$ is a skew-symmetric matrix.

The load torque is considered an unknown bounded disturbance with constant time derivative. Moreover, due to variations of both rotor and stator resistances during operations, $\alpha_{r}$ and/or $\alpha_{s}$ are assumed to be uncertain within known bounds.

Our objective is to design an adaptive observer that allows to follow time evolution of the critical machine parameters $\left(R_{r}\right.$ and/or $R_{s}$ ) as well as the rotor flux vector and the load torque.

\section{Nonlinear class of study and high gain observer design}

Generally, the dynamic behavior of IM leads to arrange it under the class of relatively fast systems. For computational issue, the high gain observer which admits an explicit correction gain, can be considered as one of the most viable candidate in the problem of state estimation. Later on, we adopt this method in our design.

Consider the nonlinear uniformly observable class of systems as the following form

$$
\left\{\begin{array}{l}
\dot{z}=f(u, z)+\varepsilon \\
y=\bar{C} z=z^{1}
\end{array}\right.
$$

where the state $z \in \mathbb{R}^{n}$ with $z^{k} \in \mathbb{R}^{n_{k}}$ for $k=1,2, \ldots, q$, and $n_{1} \geqslant n_{2} \geqslant \cdots n_{q}, \sum_{k=1}^{n} n_{k}=n$, the input $u \subset \mathbb{U}$ a compact set of $\mathbb{R}^{m}$, the output $y \in \mathbb{R}^{n_{1}}$,

$$
z=\left(\begin{array}{c}
z^{1} \\
z^{2} \\
\vdots \\
z^{q}
\end{array}\right) ; \quad f(u, z)=\left(\begin{array}{c}
f_{1}\left(u, z^{1}, z^{2}\right) \\
f_{2}\left(u, z^{1}, z^{2}, z^{3}\right) \\
\vdots \\
f_{q-1}(u, z) \\
0
\end{array}\right) ; \quad \varepsilon=\left(\begin{array}{c}
0 \\
\vdots \\
0 \\
\varepsilon^{q-1} \\
\varepsilon^{q}
\end{array}\right) ;
$$




$$
\bar{C}=\left[I_{n_{1}}, 0_{n_{1} \times n_{2}}, \ldots, 0_{n_{1} \times n_{q}}\right],
$$

with $I_{n_{1}}$ is the $n_{1} \times n_{1}$ identity matrix and $0_{n_{1} \times n_{j}}$ is the $n_{1} \times n_{j}$ null matrix, $j \in\{2, \ldots, q-1\}$.

$\varepsilon^{k} \in \mathbb{R}^{n_{k}}, k \in\{q-1, q\}$; each $\varepsilon^{k}$ is an unknown bounded real valued function that depend on uncertain parameters.

Note that this class of nonlinear systems is appropriate to the HGO design. In fact, it is sometimes easier to put the system under study into this form by using an intuitive transformation, rather than to use a more restrictive canonical form as given in [10] or [8]. Moreover, with its triangular form, the representation (2) allows to determinate step by step all the state variables (i.e. based on the knowledge of $z_{1}=y$ and its time derivative, $z_{2}$ can be extracted from the first equation of $(2)$; then $z_{3}$ can be retired from the second equation, etc.).

Now, specifying the disturbance term $\varepsilon(t)$, we propose a slight modification in relation to the model used in [9], when authors choose $\varepsilon$ with only the last component $\varepsilon^{q} \neq 0$.

The synthesis of the HGO corresponding to the form (2), requires to make some assumptions as follows:

(i) There exist $\alpha, \beta$ with $0<\alpha \leqslant \beta$ such that for all $k \in\{1, \ldots, q-1\}, \forall z \in \mathbb{R}^{n}, u \in \mathcal{U}$

$$
0<\alpha^{2} I_{n_{k}} \leqslant\left(\frac{\partial f_{k}\left(u, z^{1: k}\right)}{\partial z^{k+1}}\right)^{T} \frac{\partial f_{k}\left(u, z^{1: k}\right)}{\partial z^{k+1}} \leqslant \beta^{2} I_{n_{k}}
$$

Moreover, we assume that $\operatorname{Rank}\left(\frac{\partial f_{k}\left(u z^{1: k}\right)}{\partial z^{k+1}}\right)=n_{k+1}$.

(ii) The function $f(u, z)$ is globally Lipchitz with respect to $z$, uniformly in $u$.

(iii) The function $\varepsilon^{q-1}(t)$ and $\varepsilon^{q}(t)$ are uniformly bounded with $\delta_{q-1}=\sup _{t \geqslant 0}\left\|\varepsilon^{q-1}(t)\right\|$ and $\delta_{q}=\sup _{t \geqslant 0}\left\|\varepsilon^{q}(t)\right\|$.

By assumption (i), each of $\frac{\partial f_{k}(.)}{\partial z^{k+1}}$ is left invertible. Assumption (ii) can be omitted in the case where the trajectory $z(t)$ of system (2) lie in the bounded set $\Omega$. Whereas assumption (iii) is often physically true.

In our work, when convergence of estimation error dynamic shall be slightly modified compared to the result given in [9], we preserve the same form of the observer corresponding to model (2). Hence,

$$
\dot{\hat{z}}=f(u, \hat{z})-\theta \Lambda^{+}(\hat{z}) \Delta_{\theta}^{-1} S^{-1} C^{T} \bar{C}(\hat{z}-z)
$$

where

- $\Lambda^{+}(\hat{z})$ is the left inverse of block diagonal matrix $\Lambda(\hat{z})$ with

$$
\Lambda(\hat{z})=\operatorname{blockdiag}\left(I_{n_{1}}, \frac{\partial f_{1}\left(u, \hat{z}^{1}, \hat{z}^{2}\right)}{\partial \hat{z}^{2}}, \ldots, \prod_{i=1}^{q-1} \frac{\partial f_{k}(u, \hat{z})}{\partial \hat{z}^{k+1}}\right)
$$

- $\Delta_{\theta}=\operatorname{blockdiag}\left(I_{n_{1}}, \frac{1}{\theta} I_{n_{1}}, \ldots, \frac{1}{\theta^{q-1}} I_{n_{1}}\right)$, with $\theta>0$ is a real number representing the only design parameter of the observer.

- $S$ is a definite positive solution of the algebraic Lyapunov equation

$$
S+\mathcal{A}^{T} S+S \mathcal{A}-C^{T} C=0
$$

with

$$
\begin{aligned}
& C=\left[I_{n_{1}}, 0_{n_{1}}, \ldots, 0_{n_{1}}\right] \\
& \mathcal{A}=\left(\begin{array}{cc}
0 & \overline{\mathcal{A}} \\
0 & 0
\end{array}\right), \overline{\mathcal{A}}=\operatorname{blockdiag}\left(I_{n_{1}}, I_{n_{1}}, \ldots, I_{n_{1}}\right) \in \mathbb{R}^{n_{1}(q-1)}
\end{aligned}
$$

Note that (4) is independent of the system and the solution can be expressed analytically. For a straightforward computation, its stationary solution is given by: $S(i, j)=(-1)^{i+j} \mathcal{C}_{i+j-2}^{j-1} I_{n_{1}}$ where $\mathcal{C}_{j}^{i}=\frac{j !}{i !(j i) !}$ for $1 \leqslant i, j \leqslant q$; and then we can explicitly determinate the correction gain of (3) as follows:

$$
\theta \Lambda^{+}(\hat{z}) \Delta_{\theta}^{-1} S^{-1} C^{T}=\left(\begin{array}{c}
\theta \mathcal{C}_{1}^{q} I_{n_{1}} \\
\theta^{2} \mathcal{C}_{2}^{q}\left[\frac{\partial f_{1}}{\partial x^{2}}(u, z)\right]^{+} \\
\vdots \\
\theta^{q} \mathcal{C}_{q}^{q}\left[\prod_{i=1}^{q-1} \frac{\partial f_{k}}{\partial z^{k+1}}(u, z)\right]^{+}
\end{array}\right)
$$

It should be emphasized that the implementation of $\mathrm{HGO}$ is quite simple. 
Table 1

IM parameters used in simulations.

\begin{tabular}{llllllll}
\hline$p$ & $f(\mathrm{~Hz})$ & $L_{S}(\mathrm{H})$ & $L_{r}(\mathrm{H})$ & $M(\mathrm{H})$ & $R_{S N}(\Omega)$ & $R_{r N}(\Omega)$ & $J\left(\mathrm{~kg} \mathrm{~m}^{2}\right)$ \\
\hline 2 & 50 & 0.464 & 0.464 & 0.4417 & 5.717 & 3 & 0.0049 \\
\hline
\end{tabular}

Indeed, it requires only the tuning of the parameter $\theta$, which should be chosen as a compromise between fast convergence and satisfactory dealing with noise rejection. In practice, a convenient way to choose $\theta$ is to run a few simulations and decrease its value until a satisfactory rate of convergence for the observer is found.

Corresponding to the model class (2), the slight modification of $\varepsilon$ structure (compared to the one introduced in [9] or [8]) involves a new upper bound of the estimation $\operatorname{error}^{1}(\tilde{z}=\hat{z}-3)$ when we use the observer under form (3). The following equality holds for every $t \geqslant 0$

$$
\|\tilde{\tilde{z}}(t)\| \leqslant \lambda_{\theta} e^{-\mu_{\theta} t}\|\tilde{z}(0)\|+M_{q-1, \theta} \delta_{q-1}+M_{q, \theta} \delta
$$

with $\lambda_{\theta}=\sqrt{\frac{\lambda_{\max }(s)}{\lambda_{\min }(s)}}>0, \delta_{q-1}$ and $\delta_{q}$ (given in assumption iii) are the upper bound of $\varepsilon^{q-1}$ and $\varepsilon^{q}$ respectively. $\lim _{\theta \rightarrow \infty} \mu_{\theta}=+\infty$, $\lim _{\theta \rightarrow \infty} \mathrm{M}_{q, \theta}=0$ and $\lim _{\theta \rightarrow \infty} M_{q-1, \theta}<\mu$. A sketch of proof for estimation error convergence is given in the Appendix.

\section{Application to induction motors}

To examine practical usefulness, the proposed estimator has been simulated for a three-phase $1.5 \mathrm{kw}$ induction motor, whose parameters are depicted in Table 1. Four schemes for the real-time estimation of IM parameters and states shall be studied in the next. The first and second schemes are for separate identification of the rotor and stator resistances, while the third scheme performs simultaneous estimation of these two parameters and the fourth one deals with the rotor resistance estimation in sensorless case.

\subsection{Estimation of the rotor fluxes, the rotor resistance, and the load torque}

Here, we shall give an observer which allows to estimate the rotor resistor as well as the rotor fluxes and the load torque from angular speed and stator currents measurements $\left(R_{s}\right.$ is assumed to be known and equal to its rated value in this case). In order to synthesize a HGO as given in (3), we need to transform system (1) to the triangular form (2). Thus, let us definite the new coordinate $z$ such as

$$
\left\{\begin{array}{l}
z^{1}=\imath \\
z^{2}=A\left(\omega, \alpha_{r}\right) \Psi-\alpha_{r} M l \\
z^{3}=\alpha_{r}
\end{array}\right.
$$

Using this transformation, we can derive from model (1), a following submodel

$$
\left\{\begin{array}{l}
\dot{z}^{1}=K z^{2}-\frac{1}{\sigma} \alpha_{S N} z^{1}+\frac{1}{\sigma L_{s}} u \\
\dot{z}^{2}=-\left(z^{2}+M \dot{z}^{1}\right) z^{3}+p J_{2} z^{2} \omega+\varepsilon^{2} \\
\dot{z}^{3}=0 \\
y=\left[\left(z^{1}\right)^{T} \omega\right]^{T}
\end{array}\right.
$$

where $\varepsilon^{2}=-\dot{\omega} J_{2} \Psi$. This term shall be treated as unknown bounded function. ${ }^{2}$ We can easily show that the submodel (8) is under form (2). Hence, using Eq. (5), the HGO specializes as follows:

$$
\left\{\begin{array}{l}
\dot{\hat{z}}^{1}=K \hat{z}^{2}-\frac{1}{\sigma} \alpha_{S N} \hat{z}^{1}+\frac{1}{\sigma L_{s}} u-3 \theta_{1} \tilde{z}^{1} \\
\dot{\hat{z}}^{2}=-\left(\hat{z}^{2}+M \hat{\bar{z}}^{1}\right) \hat{z}^{3}+p J_{2} \hat{z}^{2} \omega_{r}-3 \frac{\theta_{1}^{2}}{K} \tilde{z}^{1} \\
\dot{\hat{z}}^{3}=\frac{\theta_{1}^{3}}{K}\left(\hat{z}^{2}+M \dot{\hat{z}}^{1}\right)^{+} \tilde{z}^{1}
\end{array}\right.
$$

in which $\tilde{z}^{1}=\hat{z}^{1}-z^{1}$.

Referring to (7), we can deduce an estimation of $\widehat{\Psi}$ and $\widehat{R}_{r}$ such as

$$
\widehat{\Psi}=A\left(\omega, \hat{\alpha}_{r}\right)^{-1}\left(\hat{z}^{2}+M \hat{\alpha}_{r} \hat{z}^{1}\right), \quad \widehat{R}_{r}=L_{r} \hat{\alpha}_{r}
$$

\footnotetext{
1 see Appendix.

2 This expression is assumed negligible in [14] when $\|\dot{\omega}\| \ll 2 \alpha_{r}\|\omega\|$.
} 
Please note that $A\left(\omega, \hat{\alpha}_{r}\right)$ is invertible because for $t \geqslant 0$ we have $\operatorname{det}(A)=\alpha_{r}^{2}+(p \omega)^{2} \neq 0$.

Now, rotor fluxes and rotor resistance provided by (9) are used to determinate the load torque $\widehat{T}_{L}$ and its time derivative $\widehat{T}_{L p}$. In fact, we have

$$
\left\{\begin{array}{l}
\dot{\hat{\omega}}=-\frac{1}{J} \widehat{T}_{L}+\frac{p M}{J L_{r}} \hat{l}^{T} J_{2}\left(\hat{\alpha}_{r} I_{2}-p J_{2} \hat{\omega}\right)^{-1} \hat{z}^{2}-3 \theta_{2} \tilde{\omega} \\
\dot{\widehat{T}}_{L}=\widehat{T}_{L p}+3 \theta_{2}^{2} J \tilde{\omega} \\
\hat{\widehat{T}}_{L p}=\theta_{2}^{3} J \tilde{\omega}
\end{array}\right.
$$

where $\tilde{\omega}=\hat{\omega}-\omega$.

In order to evaluate the observer behavior in the realistic situation, the measurements of $y$ issued from the model simulation have been corrupted by noise measurements with a zero mean value and a variance $\sigma_{n}=10^{-4}$. The parameters design of the two-stage HGO are chosen as $\theta_{1}=700$ and $\theta_{2}=200$. We suggest a trapezoidal rising profile of the rotor resistance up to $100 \%$ of its rated value (which in practical way can be obtained by using 3-phase variable rheostat for rotor wound IM). The dynamic behavior of the rotor speed is depicted in Fig. 1 graph (a); when graph (b) and graph (c) shows the estimation of both load torque and rotor resistance. Despite some fluctuations at the transient, a good agreement between actual and estimated states is achieved in each case. Indeed, when permanent regime is established the annulation of the angular speed dynamics $(\dot{\omega}=0)$ involves an annulation of the uncertainties $\left(\varepsilon^{2}=0\right)$. Thus, by referring to $(6)$ an exponential convergence of the estimation error is achieved. Note that, a little bounded error can be seen when $\omega(t)$ is time varying, it is caused by the fact that $\varepsilon^{2} \neq 0$. Moreover, the capability of proposed algorithm to estimate parameter fault is investigated by simulation of a short circuit fault for $t \geqslant 1.15 \mathrm{~s}$. The satisfactory tracking result of the rotor resistance corresponding to this abrupt variation brings to our design the privilege that can be used in the fault detection issue, and thereafter ensuring safe operation modes. In other hand and reported to Fig. 2, we remark a quite performance in the estimation of the rotor flux modulus and its both components in the fixed reference frame $(\alpha, \beta)$.

\subsection{Estimation of the rotor fluxes, the stator resistance, and the load torque}

Now, instead of the rotor resistance, we propose a new coordinate transformation that allows an estimation of the stator resistance which presents also a critical parameter in IMs. We assume that the rotor resistance keep its rated value $R_{r N}$ in this case. Let us adopt the new state variables as follows:

(a)

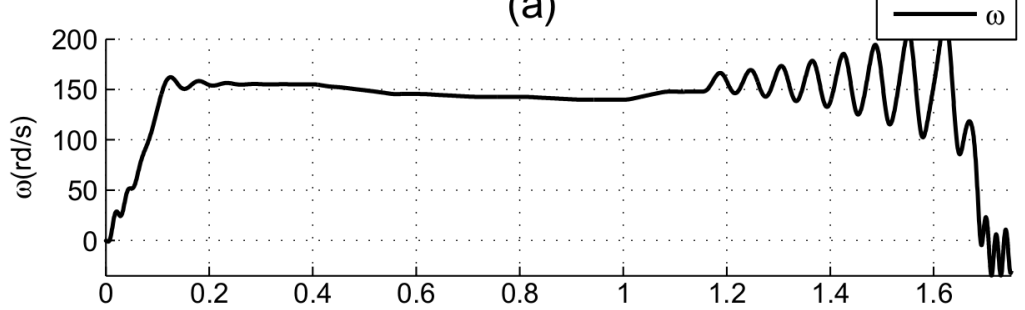

(b)

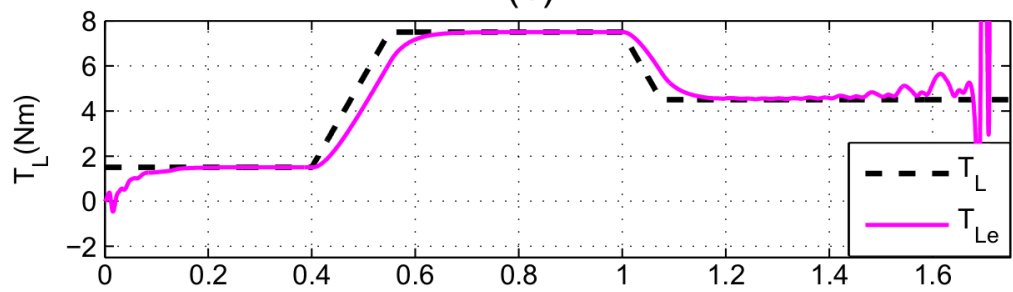

(c)

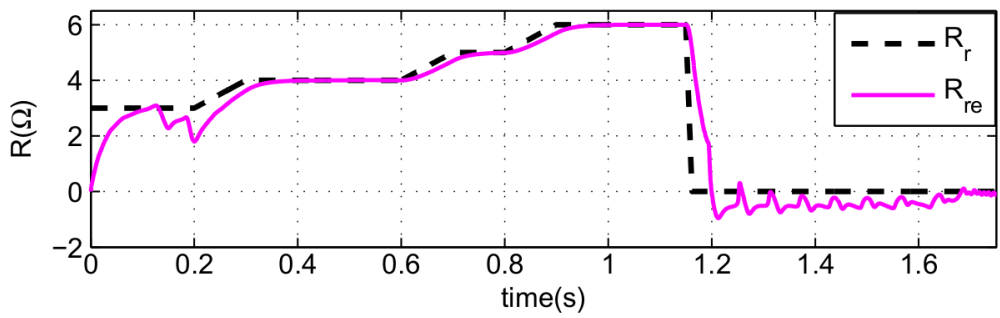

Fig. 1. Simulation results for the open loop study. (a) Rotor speed response. (b) Load torque estimation. (c) Rotor resistance update. 

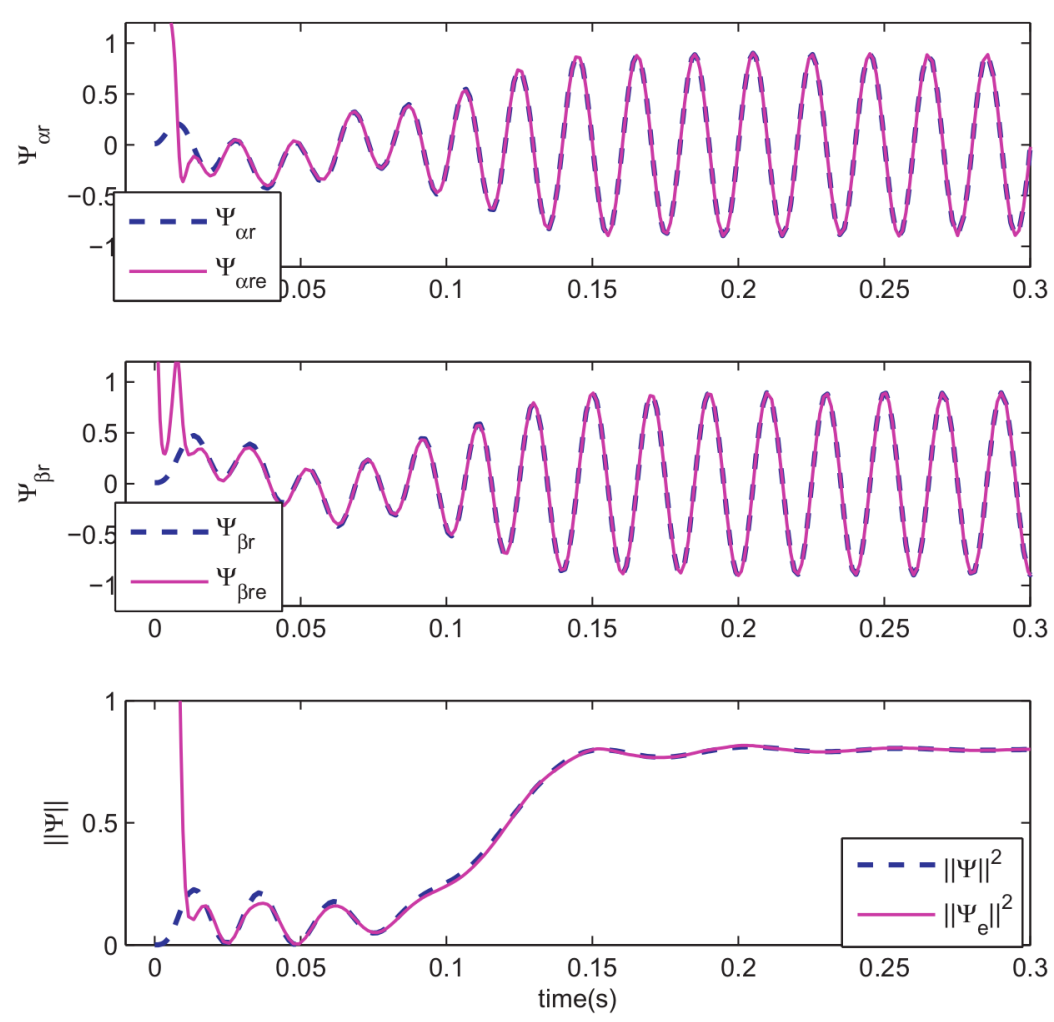

Fig. 2. The rotor fluxes estimation.

$$
\left\{\begin{array}{l}
z^{1}=l \\
z^{2}=K A(\omega) \Psi-\frac{1}{\sigma} \alpha_{s} l \\
z^{3}=\alpha_{s}
\end{array}\right.
$$

a time derivative of these states leads to

$$
\left\{\begin{array}{l}
\dot{z}^{1}=z^{2}-K M \alpha_{r N} z^{1}+\frac{1}{\sigma L_{s}} u \\
\dot{z}^{2}=-\frac{1}{\sigma}\left(A(\omega) z^{1}+\dot{z}^{1}\right) z^{3}-A(\omega) z^{2}+K M \alpha_{r N} A(\omega) z^{1}+\varepsilon^{2} \\
\dot{z}^{3}=0 \\
y=\left[\left(z^{1}\right)^{T} \omega\right]^{T}
\end{array}\right.
$$

where $\varepsilon^{2}$ is given as above. The corresponding HGO admits the following form

$$
\left\{\begin{array}{l}
\dot{\hat{z}}^{1}=\hat{z}^{2}-K M \alpha_{r N} \hat{z}^{1}+\frac{1}{\sigma L_{s}} u-3 \theta_{1} \tilde{z}^{1} \\
\dot{\hat{z}}^{2}=-\frac{1}{\sigma}\left(A(\omega) \hat{z}^{1}+\dot{\hat{z}}^{1}\right) \hat{z}^{3}-A(\omega) \hat{z}^{2}+K M \alpha_{r N} A(\omega) \hat{z}^{1}-3 \theta_{1}^{2} \tilde{z}^{1} \\
\dot{\hat{z}}^{3}=\theta_{1}^{3} \sigma\left(A(\omega) \hat{z}^{1}+\dot{\hat{z}}^{1}\right)^{+} \tilde{z}^{1}
\end{array}\right.
$$

Referring to (11), the rotor fluxes and the stator resistance are governed by the following equations

$$
\widehat{\Psi}=\frac{1}{K} A(\omega)^{-1}\left(\hat{z}^{2}+\frac{1}{\sigma} \hat{z}^{1} \hat{\alpha}_{s}\right), \quad \widehat{R}_{s}=L_{s} \hat{\alpha}_{s}
$$

As previously, an estimation of the load torque $\widehat{T}_{L}$ and its time derivative $\widehat{T}_{L p}$ can be given by (10) when $\alpha_{r N}$ replace $\hat{\alpha}_{r}$. The frequency is controlled by a predictive control law [20]. Estimation results of the proposed algorithm with $\theta_{1}=850$ and $\theta_{2}=100$ are reported in Figs. (3) and (4). Both graphs (b) and (c) of Fig. 3 correspond to the estimation of the time varying load torque and the stator resistance which increases up to $80 \%$ of its rated value. Note that, in spite of the large initial estimation error, the obtained estimate of the load torque quickly converge to the actual value. We show also that there are little fluctuations in the stator resistance estimation even at low frequency operations, they are caused by the term of uncertainty $\varepsilon^{2}$ which affects the convergence properties when $\dot{\omega} \neq 0$. The rotor fluxes estimation are illustrated in Fig. (4). As it can be seen, a rallying behavior between the guess and the true values is nearly achieved. 
(a)

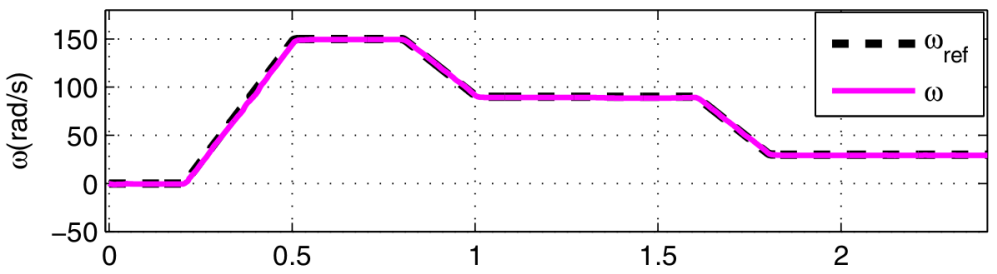

(b)

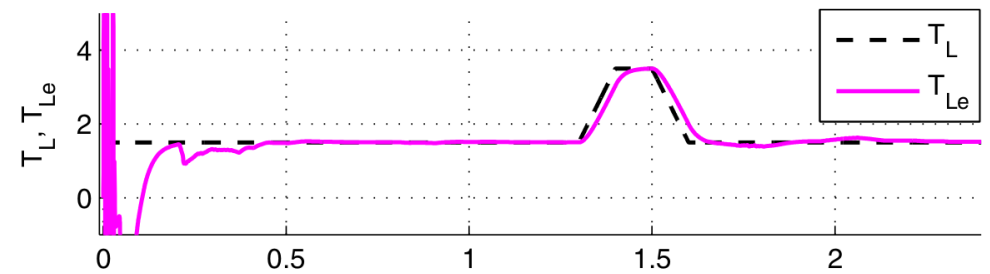

(c)

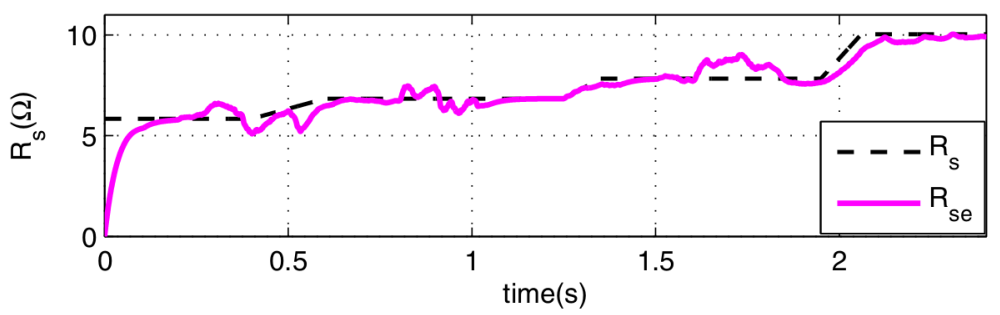

Fig. 3. High performance tracking reference for the mechanical speed (a), based on load torque estimation (b) and stator resistance update (c).
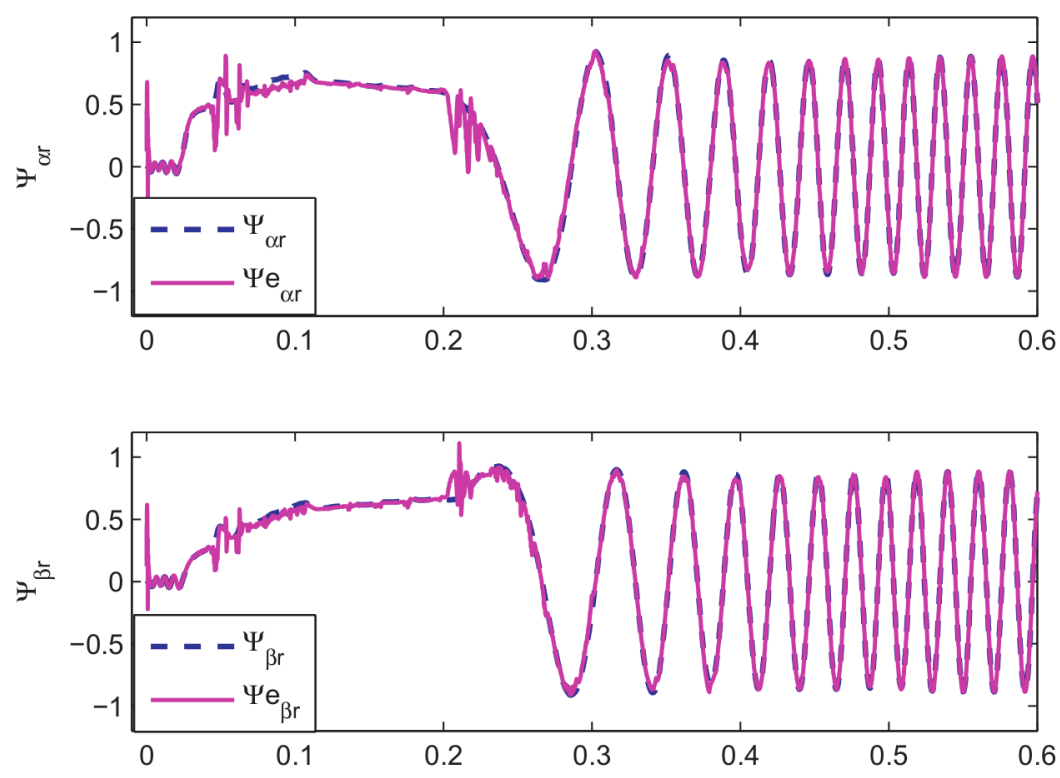

Fig. 4. The rotor fluxes estimation.

4.3. Estimation of the rotor fluxes, both rotor and stator resistances, and the load torque

In order to simultaneously estimate both rotor and stator resistances, we use another state transformation such as model (1) can be rewritten again under form (2). When we choose 


$$
\left\{\begin{array}{l}
z^{1}=l \\
z^{2}=K\left(A\left(\omega, \alpha_{r}\right) \Psi-\alpha_{r} M l\right)-\frac{1}{\sigma} \alpha_{s} l \\
z^{3}=\left[\alpha_{s} \alpha_{r}\right]^{T}
\end{array}\right.
$$

We obtain the following submodel

$$
\left\{\begin{array}{l}
\dot{z}^{1}=z^{2}+\frac{1}{\sigma L_{s}} u \\
\dot{z}^{2}=-\frac{1}{\sigma}\left(\dot{z}^{1}+A\left(\omega, \alpha_{r}\right) z^{1}\right) \alpha_{s}-\left(K M \dot{z}^{1}\right) \alpha_{r} \\
-A\left(\omega, \alpha_{r}\right) z^{2}+\varepsilon^{2} \\
\dot{z}^{3}=0 \\
y=\left[\left(z^{1}\right)^{T} \omega\right]^{T}
\end{array}\right.
$$

So, a candidate observer is given by

$$
\left\{\begin{array}{l}
\dot{\hat{z}}^{1}=\hat{z}^{2}+\frac{1}{\sigma L_{s}} u-3 \theta_{1} \tilde{z}^{1} \\
\dot{\vec{z}}^{2}=-\frac{1}{\sigma}\left(\dot{\bar{z}}^{1}+A\left(\omega, \hat{\alpha}_{r}\right) \hat{z}^{1}\right) \hat{\alpha}_{s}-\left(K M \dot{\bar{z}}^{1}\right) \hat{\alpha}_{r} \\
-A\left(\omega, \hat{\alpha}_{r}\right) \hat{z}^{2}-3 \theta_{1}^{2} \tilde{z}^{1} \\
\dot{\hat{z}}^{3}=-\theta_{1}^{3} \Lambda^{-1} \tilde{z}^{1}
\end{array}\right.
$$

(a)

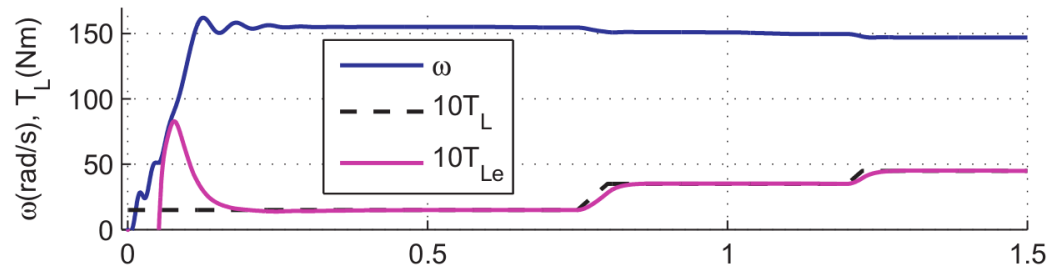

(b)

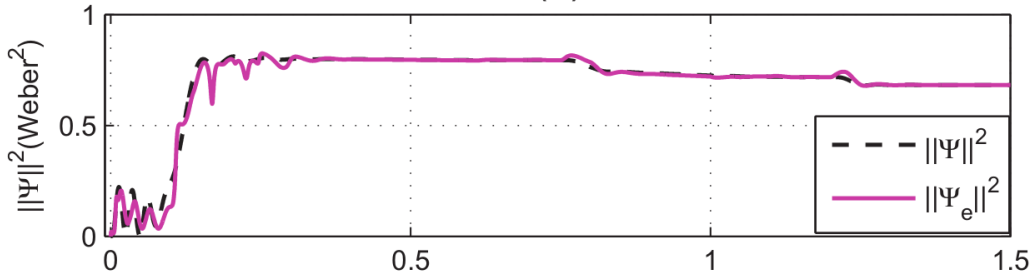

(c)

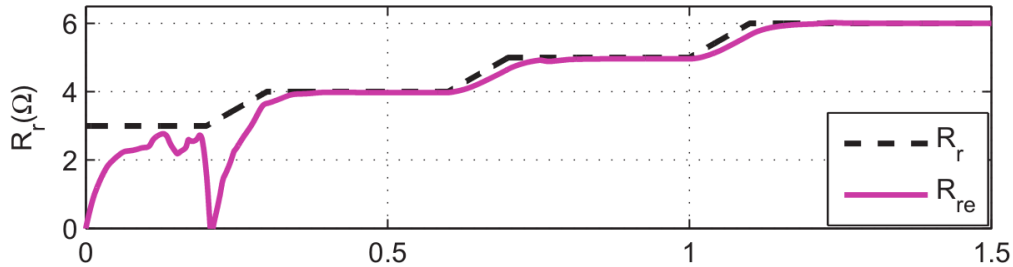

(d)

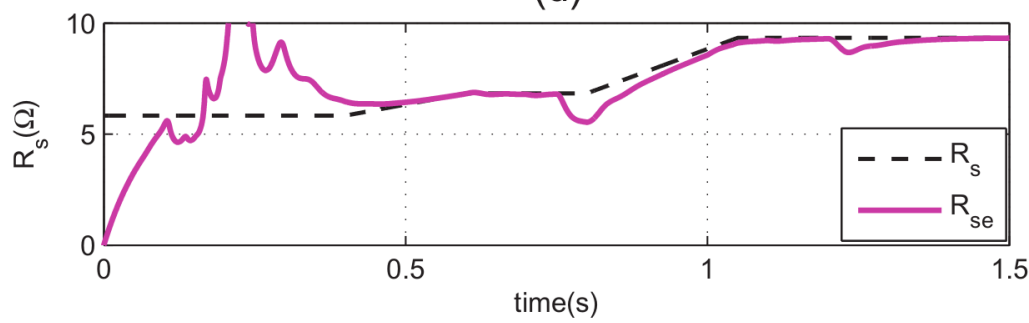

Fig. 5. Open loop estimation of the rotor flux modulus, the load torque and both rotor and stator resistances. 
with

$$
\Lambda=\left(\begin{array}{c}
-\frac{1}{\sigma}\left(\dot{\hat{z}}^{1}-p \omega J_{2} \hat{z}^{1}+\hat{z}^{1} \hat{\alpha}_{r}\right)^{T} \\
-\left(K M \dot{\hat{z}^{1}}+\hat{z}^{2}+\frac{1}{\sigma} \hat{z}^{1} \hat{\alpha}_{s}\right)^{T}
\end{array}\right)
$$

Referring to (14), we can deduce an estimation of the rotor flux vector and both rotor and stator resistances such as

$$
\left\{\begin{array}{l}
\widehat{\Psi}=A\left(\omega, \hat{\alpha}_{r}\right)^{-1}\left(\frac{1}{K}\left(\hat{z}_{2}+\frac{1}{\sigma} \hat{z}_{1} \hat{\alpha}_{s}\right)+M \hat{\alpha}_{r} \hat{z}_{1}\right) \\
\widehat{R}_{r}=L_{r} \hat{\alpha}_{r} \\
\widehat{R}_{s}=L_{s} \hat{\alpha}_{s}
\end{array}\right.
$$

The load torque $\widehat{T}_{L}$ and its time derivative $\widehat{T}_{L p}$ can arise, as previously, from Eq. (10).

To show the merits of the proposed observation scheme, an arbitrary rising trapezoidal profiles are suggested in our simulation to the load torque and both rotor and stator resistances.

Fig. (5) presents the performance attained with $\theta_{1}=1200$ and $\theta_{2}=100$. In spite of some overshoots in start-up, graphs (a) and (c) indicate that the convergence of the estimates $\widehat{R}_{r}$ and $\widehat{T}_{L}$ to their trues values is achieved in the operation range. Graph (b) demonstrates also a quite reconstruction of the rotor flux modulus, when graph (d) illustrates a sensibility of the stator resistance estimation to the load torque variation, and consequently to the transient operating mode.

\subsection{Estimation of the rotor fluxes, rotor resistance and the load torque for speed sensorless IMs}

One of the main actual challenges in IM is to consider an estimation of the rotor resistance without using a digital shaft speed or position sensor. Now, we shall synthesize an observer that allows to estimate the rotor fluxes and the mechanical speed as well as the load torque and the rotor resistor from only stator currents measurements.

(a)

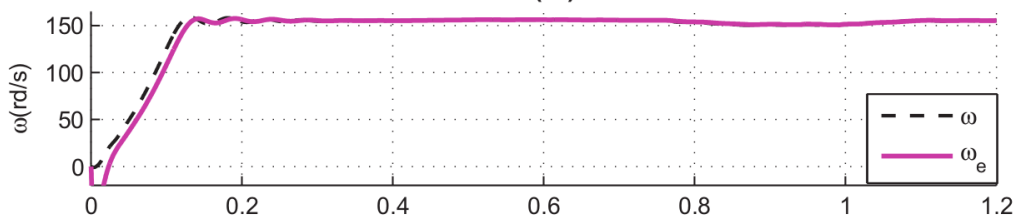

(b)

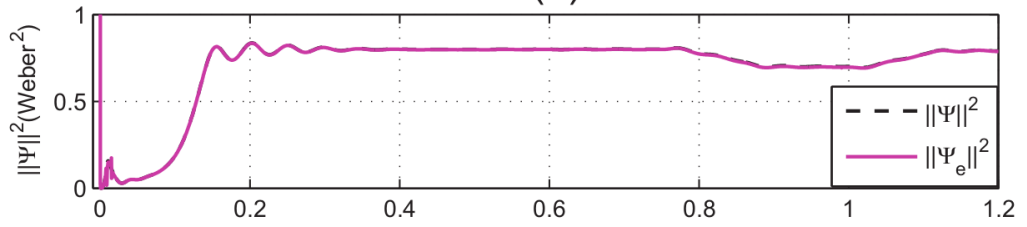

(c)

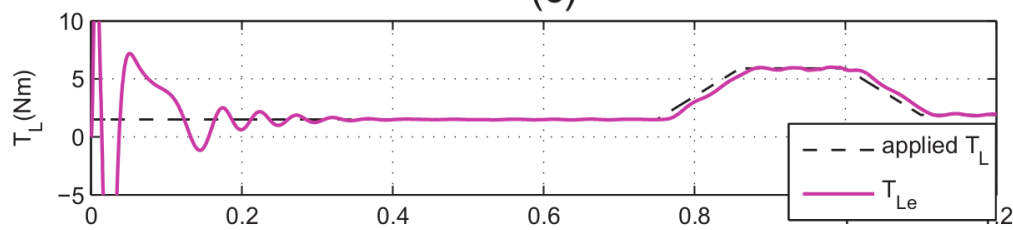

(d)

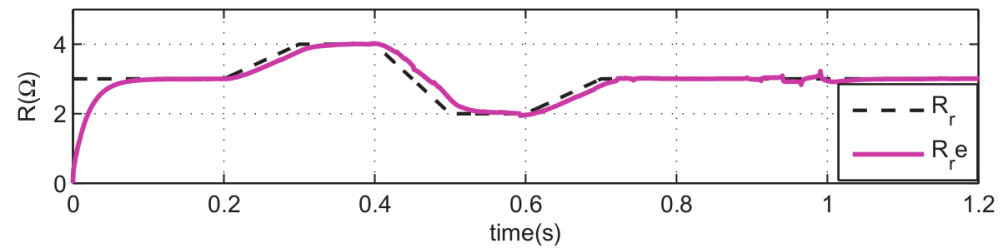

Fig. 6. Open loop estimation of the angular speed, the load torque and the rotor resistance. 
Let us definite the new coordinates $z$ such as

$$
\left\{\begin{array}{l}
z^{1}=\imath \\
z^{2}=A\left(\omega, \alpha_{r}\right) \Psi-\alpha_{r} M \imath \\
z^{3}=\left[\begin{array}{ll}
\omega & \alpha_{r}
\end{array}\right]^{T}
\end{array}\right.
$$

Consequently, we have

$$
\left\{\begin{array}{l}
\dot{z}^{1}=K z^{2}-\frac{R_{s}}{\sigma L_{s}} z^{1}+\frac{1}{\sigma L_{s}} u \\
\dot{z}^{2}=p J_{2} z^{2} z_{1}^{3}-\left(M \dot{z}^{1}+z^{2}\right) z_{2}^{3}-p J_{2} \Psi^{\star} d \omega+\varepsilon^{2} \\
\dot{z}^{3}=\left[d \omega+\varepsilon_{1}^{3} 0\right]^{T} \\
y=z^{1}
\end{array}\right.
$$

with $d \omega=\frac{p M}{J L_{r}} z^{1^{T}} J_{2} \Psi^{\star}, \Psi^{\star}=A(s)^{-1}\left(z^{2}+\alpha_{r} M z^{1}\right), \varepsilon^{2}=\frac{p}{J} J_{2} \Psi^{\star} T_{L}$ and $\varepsilon_{1}^{3}=-\frac{1}{J} T_{L}$. Last both terms shall be treated as unknown bounded functions.

Thereafter, we can synthesize the following observer

$$
\left\{\begin{array}{l}
\dot{z}^{1}=K \hat{z}^{2}-\frac{R_{s}}{\sigma L_{s}} \hat{z}^{1}+\frac{1}{\sigma L_{s}} u-3 \theta_{1} \tilde{z}^{1} \\
\dot{z}^{2}=p J_{2} \hat{z}^{2} \hat{z}_{1}^{3}-\left(M \hat{z}^{1}+\hat{z}^{2}\right) \hat{z}_{2}^{3}-p J_{2} \widehat{\Psi}^{\star} \widehat{d \omega}-3 \frac{\theta_{1}^{2}}{K} \tilde{z}^{1} \\
\dot{z}^{3}=[\widehat{d \omega} 0]^{T}-\frac{\theta_{1}^{3}}{K} \Lambda^{+}(\hat{z}) \tilde{z}^{1}
\end{array}\right.
$$

in which $\tilde{z}^{1}=\hat{z}^{1}-z^{1}, \Lambda(\hat{z})=\left[\frac{\partial \dot{z}^{2}}{\partial \hat{z}^{3}}\right]_{\theta_{1}=0}=\left[p J_{2} \hat{z}^{2}-\left(M \dot{\hat{z}}^{1}+\hat{z}^{2}\right)\right]$.

Referring to (18), it is easy to seen that we can deduce an estimation of $\widehat{\Psi}, \hat{\omega}$ and $\widehat{R}_{r}$ as follows:

$$
\left\{\begin{array}{l}
\hat{\omega}=\hat{z}_{1}^{3} \\
\widehat{R}_{r}=L_{r} \hat{z}_{2}^{3} \\
\widehat{\Psi}=\left(\hat{z}_{2}^{3} I_{2}-p J_{2} \hat{z}_{1}^{3}\right)^{-1}\left(\hat{z}^{2}+\hat{z}_{2}^{3} M \hat{z}^{1}\right)
\end{array}\right.
$$

As previously, the rotor fluxes and the mechanical speed estimates provided by (20) and (21) are used to reconstruct the load torque $\widehat{T}_{L}$ and its time derivative $\widehat{T}_{L p}$.

Fig. (6) shows the estimation results of the proposed observer with $\theta_{1}=2000$ and $\theta_{2}=1250$. In graphs (a) and (b) the angular speed and the rotor flux modulus are well reconstructed. The rotor resistance estimation illustrated in graph (d) is slightly influenced by the load torque which converges to its true value after the transient regime (graph (c)).

\section{Conclusion}

In this paper, an alternative form for a high gain observer is presented. It allows to conjointly estimate state variables and time varying parameters. This strategy helps not only, to on-line follow time evolution of critical parameters and state variables; but also, to detect some appropriate fault for a given normal operating range. The satisfactory results given for an application to the induction motors, confirm the good behavior of the proposed algorithm to track the mismatch of both rotor and stator resistances as well as the time evolution of the rotor flux and the load torque; and therefore the possible exploitation of our design in the fault detection issue (i.e. short-circuit detection when $\widehat{R}_{r} \simeq 0$ ). The efficiency in real time computation can be once more highlight when the proposed observer ensure the convergence properties at zero speed which remains a challenge. Thus, our futur work will focus on the resolution of this open problem.

\section{Appendix A}

Proceeding as in [9], we can show that the transformation

$$
\Phi_{0}: \mathbb{R}^{n} \rightarrow \mathbb{R}^{n_{1} q}, \quad z \mapsto \mathfrak{z}=\Phi_{0}(z)=\left(\begin{array}{c}
z^{1} \\
f_{1}\left(u, z^{1}, z^{2}\right) \\
\frac{\partial f_{1}(\cdot)}{\partial z^{2}} f_{2}\left(u, z^{1}, z^{2}, z^{3}\right) \\
\vdots \\
\left(\prod_{i=1}^{q-2} \frac{\partial f_{k}(\cdot)}{\partial z^{k+1}}\right) f_{q-1}(u, z)
\end{array}\right)
$$

puts system (2) under the following form

$$
\left\{\begin{array}{l}
\dot{\mathfrak{z}}=\mathcal{A}_{\mathfrak{z}}+\phi(u, \mathfrak{3})+\frac{\partial \Phi_{0}}{\partial \mathfrak{z}} \varepsilon \\
y=C_{\mathfrak{z}}=\mathfrak{z}^{1}
\end{array}\right.
$$


with $\phi(u, \mathfrak{\jmath})$ has a triangular structure and $C$ is as given above. Let consider a HGO design for the system (A.1) as follows [8]:

$$
\dot{\hat{\mathfrak{z}}}=\mathcal{A} \hat{\mathfrak{z}}+\phi(u, \hat{\mathfrak{z}})-\theta \Delta_{\theta}^{-1} S^{-1} C^{T} \tilde{\mathfrak{z}}^{1}-\theta \Gamma(u, \hat{\mathfrak{z}}) \Delta_{\theta}^{-1} S^{-1} C^{T} \tilde{\mathfrak{z}}^{1}
$$

where

$$
\Gamma(u, \hat{\jmath})=\frac{\partial \Phi_{0}\left(u, \Phi_{0}^{c}(\hat{\mathrm{z}})\right)}{\partial z}\left(\Lambda^{+}\left(u, \Phi_{0}^{c}(\hat{\mathrm{z}})\right)-\left(\frac{\partial \Phi_{0}\left(u, \Phi_{0}^{c}(\hat{\mathrm{z}})\right)}{\partial z}\right)^{+}\right), \tilde{\jmath}^{1}=C \hat{\mathrm{z}}-y
$$

Notice that $\Gamma(u, \hat{\jmath})$ is a lower triangular matrix with zeros in its main diagonal. Moreover, using assumptions (i) and (iii), one can easily deduce that $\Gamma(u, \hat{z})$ is bounded.

Such as $\theta \Delta_{\theta}^{-1} A \Delta_{\theta}=A$ and $C \Delta_{\theta}=C$, when we set $\overline{\mathfrak{z}}=\Delta_{\theta} \tilde{z}$, we obtain

$$
\dot{\overline{\mathfrak{z}}}=\theta\left(\mathcal{A}-S^{-1} C^{T} C\right) \overline{\mathfrak{z}}+\Delta_{\theta}(\phi(u, \hat{\mathfrak{z}})-\phi(u, \mathfrak{\jmath}))-\Delta_{\theta} \frac{\partial \Phi_{0}}{\partial z} \varepsilon-\theta \Delta_{\theta} \Gamma(u, \hat{\jmath}) \Delta_{\theta}^{-1} C^{T} \tilde{\mathfrak{z}}^{1}
$$

Consider the quadratic function $V_{1}(\overline{\bar{a}})=\overline{\bar{b}}^{T} S_{\overline{3}}$, then proceeding as in [9], we have

$$
\dot{V}_{1} \leqslant-\theta V_{1}+2 \sigma^{2}(S)\|\bar{\partial}\|\left(\zeta\|\bar{\jmath}\|+\frac{\rho}{\lambda_{\min (S)}}\left\|\tilde{\jmath}^{1}\right\|\right)+\left\|\Lambda_{\theta} \frac{\partial \Phi_{0}\left(u, \Phi_{0}^{c}(\hat{\jmath})\right)}{\partial z} \varepsilon\right\|
$$

where $\sigma(S)=\sqrt{\frac{\lambda_{\max }(S)}{\lambda_{\min }(S)}}$ with $\lambda_{\min }(S)$ and $\lambda_{\max }(s)$ being respectively the smallest and the largest eigenvalues of matrix $S . \zeta$ and $\rho$ are respectively the upper bound of $\frac{\left\|\Delta_{\theta}\left(\phi(u, \hat{)})-\phi\left(u_{\hat{z}}\right)\right)\right\|}{\|\vec{z}\|}$ and $\left\|\theta \Delta_{\theta} \Gamma(u, \hat{\jmath}) \Delta_{\theta}^{-1}\right\|$.

According to the $\varepsilon$ structure and since $\frac{\partial \Phi_{0}\left(u \Phi_{0}^{c}(\hat{z})\right)}{\partial z}$ is triangular, it follows that

$$
\begin{aligned}
\left\|\Delta_{\theta} \frac{\partial \Phi_{0}\left(u, \Phi_{0}^{c}(\hat{z})\right)}{\partial z} \bar{\varepsilon}\right\|= & \frac{1}{\theta^{q-1}} \| \theta \prod_{i=1}^{q-2}\left(\frac{\partial f^{k}(u, z)}{\partial z^{k+1}}\right) \varepsilon^{q-1} \\
& +\frac{\partial\left(\prod_{i=1}^{q-1}\left(\frac{\partial f^{k}(u, z)}{\partial z^{k}}\right) f^{q-1}\right)}{\partial z^{q-1}} \varepsilon^{q-1}+\prod_{i=1}^{q-1}\left(\frac{\partial f^{k}(u, z)}{\partial z^{k+1}}\right) \varepsilon^{q} \| \\
& \leqslant \frac{1}{\theta^{q-1}}\left(\theta \beta^{q-2}\left\|\varepsilon^{q-1}\right\|+v\left\|\varepsilon^{q-1}\right\|+\beta^{q-1}\left\|\varepsilon^{q-1}\right\|\right) \\
& \leqslant \frac{1}{\theta^{q-1}}\left(\theta \beta^{q-2} \delta^{q-1}+v \delta^{q-1}+\beta^{q-1} \delta^{q}\right) \\
& \leqslant \frac{1}{\theta^{q-1}}\left(\theta \beta^{q-2} \delta^{q-1}+\left(v+\beta^{q-1}\right) \delta\right)
\end{aligned}
$$

where $v=\sup \left(\partial\left(\prod_{i=1}^{q-1}\left(\frac{\partial f^{k}(u, z)}{\partial z^{k}}\right) f^{q-1}\right) / \partial z^{q-1}\right), k=1, \ldots, q-1$; and $\delta=\max \left(\delta^{q-1}, \delta^{q}\right)$, with $\delta^{q-1}, \delta^{q}$ and $\beta$ are given respectively in assumptions (iii) and (i). Consequently

$$
\dot{V}_{1} \leqslant-\left(\theta-c_{1}\right) V_{1}+\frac{c_{2}}{\theta^{q-1}} \delta_{\theta} \sqrt{V_{1}}
$$

where $\delta_{\theta}=\theta \beta^{q-2} \delta_{q-1}+\left(v+\beta^{q-1}\right) \delta, c_{1}=2 \sigma^{2}(S)\left(\zeta+\frac{\gamma}{\lambda_{\min (S)}}\right)$ and $c_{2}=2 \sigma(S) \sqrt{\lambda_{\max }(S)}$.

On the other hand, for $\theta \geqslant 1$, we have $\|\overline{\mathfrak{z}}(t)\| \leqslant\|\tilde{\mathfrak{z}}(t)\| \leqslant \theta^{q-1}\|\overline{\mathfrak{z}}(t)\|$, we have

$$
\|\tilde{z}(t)\| \leqslant \theta^{q-1} \sigma(S) e^{-\left(\frac{\theta-c_{1}}{2}\right) t}\|\tilde{\tilde{z}}(0)\|+2 \frac{\sigma^{2}(S)}{\theta-c_{1}}\left(\theta \beta^{q-2} \delta_{q-1}+\left(v+\beta^{q-1}\right) \delta\right)
$$

Hence, to obtain (6), we can easily define that: $\lambda_{\theta}=\theta^{q-1} \sigma(S), \mu_{\theta}=\frac{\theta-c_{1}}{2}, M_{q-1, \theta}=2 \frac{\theta}{\theta-c_{1}} \sigma^{2}(S) \beta^{q-2}$ and $M_{q, \theta}=2 \frac{1}{\theta-c_{1}} \sigma^{2}(S)(v+$ $\left.\beta^{q-1}\right)$. Finally, in the original coordinates, we can rewrite the equation of the observer as follows:

$$
\dot{\hat{z}}=\left(\frac{\partial \Phi(\hat{z})}{\partial z}\right)^{+}(\dot{\hat{\jmath}})=f(u, \hat{z})-\theta \Lambda^{+}(\hat{z}) \Delta_{\theta}^{-1} S^{-1} C^{T} \bar{C}(\hat{z}-z)
$$

\section{References}

[1] T. Ahmed-Ali, R. Postoyan, F. Lamnabhi-Lagarrigue, Continuous-discrete adaptive observers for state affine systems, Automatica 45 (2009) $2986-2990$.

[2] T. Alexandru, G. Besancon, Simultaneous state and parameter estimation in asynchronous motors under sensorless speed control, in: ECC, Orlando USA, 2003.

[3] G. Bartolini, A. Pisano, P. Pisu, Simplified exponentially convergent rotor resistance estimation for induction motors, IEEE Transactions on Automatic Control 48 (2003) 325-330.

[4] M. Barut, M.G.S. Bogosyan, Speed sensorless direct torque control of ims with rotor resistance estimation, Energy Conversion and Management 46 (2005) 335-349. 
[5] M. Barut, M.G.S. Bogosyan, Switching ekf technique for rotor and stator resistance estimation in speed sensorless control of ims, Energy Conversion and Management 48 (2007) 3120-3134.

[6] R. Beguenane, M.A. Ouhrouche, A.M. Trzynadlowski, A new scheme for sensorless induction motor control drives operating in low speed region, Mathematics and Computers in Simulation 71 (2006) 109-120.

[7] M. Farza, M. M'Saad, T. Maatoug, Adaptive observers for nonlinearly parameterized class of nonlinear systems, Automatica 45 (2009) $2292-2299$.

[8] M. Farza, M. M'Saad, L. Rossignol, Observer design for a class of mimo nonlinear systems, Automatica 40 (2004) 135-143.

[9] M. Farza, M. M'Saad, M. Sekher, A set of observers for a class of nonlinear systems, in: 16th IFAC W. C., Czech R., 2005.

[10] J. Gauthier, H. Hammouri, S. Othman, A simple observer for nonlinear systems, IEEE Transactions on Automatic Control 37 (1992) 875-880.

[11] M. Ghanes, J. Barbot, J.D. Leon, A. Glumineau, A robust output feedback controller of the induction motor drives: new design and experimental validation, International Journal of Control 83 (2010) 484-497.

[12] M. Ghanes, G. Zheng, On sensorless induction motor drives: sliding-mode observer and output feedback controller, IEEE Transactions on Industrial Electronics 56 (2009) 3404-3413.

[13] S. Jeon, K. Oh, J. Choi, Flux observer with online tuning of stator and rotor resistances for induction motors, IEEE Transactions on Industrial Electronics 49 (2002) 653-663.

[14] G. Kenne, A.b. Tarek, F. Lamnabhi-Lagarriguec, A. Arzande, Nonlinear systems time-varying parameter estimation: application to induction motors, Electric Power Systems Research 78 (2008) 1881-1888

[15] H.M. Kojabadi, Active power and mras based rotor resistance identification of an im drive, Simulation Modelling Practice and Theory 17 (2009) $376-$ 389.

[16] Y. Koubaa, M. Boussak, Rotor resistance tuning for indirect stator flux oriented induction motor drive based on mras scheme, European Transactions on Electrical Power 15 (2005) 557-570.

[17] M. Li, J. Chiasson, M. Bodson, L.M. Tolbert, A differential-algebraic approach to speed estimation in an induction motor, IEEE Transactions on Automatic Control 51 (7) (2006) 1172-1177.

[18] R. Marino, P. Tomei, S. Peresada, On-line stator and rotor resistance estimation for induction motors, IEEE Transactions on Control Systems Technology 8 (2000) $570-579$

[19] R. Marino, P. Tomei, C. Verrelli, An adaptive tracking control from current measurements for induction motors with uncertain load torque and rotor resistance, Automatica 44 (2008) 2593-2599.

[20] S.H. Saïd, N.B. Nasr, F. Mimouni, F. M'Sahli, Output feedback predictive controller for a class of nonlinear systems, in: IEEE ACC, Baltimore, USA, 2010.

[21] P.R. Sanchez, A.G. Cerradab, V. Batllea, Rotor-resistance estimation for induction machines with indirect-field orientation, Control Engineering Practice 48 (2007) 1119-1133.

[22] H.A. Toliyat, E. Levi, M. Raina, A review of rfo induction motor parameter estimation techniques, IEEE Transactions on Energy Conversion 18 (2003) $271-283$. 